\title{
A MULTIDISCIPLINARY APPROACH TO BREAST CANCER - INTRODUCING A MANAGEMENT FILE FOR BREAST CANCER PATIENTS
}

\author{
Alexandra C. BACIU ${ }^{1 凶}$, Olivia A. MARCU ${ }^{2}$, Marius S. POP ${ }^{3}$, Daiana DEBUCEAN ${ }^{3}$ \\ ${ }^{1}$ Beauty Concept Clinic, Cluj-Napoca, Romania \\ ${ }^{2}$ Faculty of Medicine and Pharmacy, University of Oradea, Romania \\ ${ }^{3}$ Linea Medica Clinic, Oradea, Romania
}

Received 18 Jan 2020, Accepted 15 Febr 2020

hitps://doi.org/10.31688/ABMU.2020.55.1.09

\begin{abstract}
Introduction. The increasing incidence of the breast cancer needs more comprehensive assessment approaches to ensure the best quality of the medical care before and after mastectomy and breast reconstruction, to minimize the negative consequences and to target maximum therapeutic compliance. The best results may be achieved in a multidisciplinary team.

The objective of the study was to introduce the Multidisciplinary Management File for Breast Cancer Patients, for a more comprehensive management of the breast cancer case.
\end{abstract}

Material and methods. Twenty patients of this prospective study, aged 32-65 years, divided into 2 groups: the study group (10 patients) in which the Multidisciplinary Management File for Breast Cancer Patients was used and the control group (10 patients), in which the classic approach was applied.

Results. The openness for the multidisciplinary approach was increased in persons living in urban areas (80\%) and with higher education $(90 \%$ high school graduates or more). Unknown pathologies were detected: 3 thyroid diseases, 1 case of diabetes mellitus,

\section{Résumé}

Approche multidisciplinaire du cancer du sein - introduction d'un fichier de gestion pour les patients avec cancer du sein

Introduction. L'incidence croissante du cancer du sein nécessite des approches d'évaluation plus complètes afin d'assurer la meilleure qualité de soins médicaux avant et après la mastectomie et la reconstruction mammaire, pour minimiser les conséquences négatives et viser une observance thérapeutique maximale. Les meilleurs résultats peuvent être obtenus dans une équipe multidisciplinaire.

Objectif. Présenter la Carte de gestion multidisciplinaire pour les patientes atteintes d'un cancer du sein, dans le but d'une prise en charge plus complète du cancer du sein.

Méthodes. 20 patientes de cette étude prospective, âgées de 32 à 65 ans, ont été réparties en 2 groupes; le groupe d'étude (10 patientes) dans lequel la Carte de gestion multidisciplinaire pour les patientes atteintes d'un cancer du sein a été utilisée et le groupe témoin (10 patientes), dans lequel l'approche classique était appliquée. 
6 patients with postural changes, biomechanical alterations of the scapular belt, muscle compensations, anxiety (100\%) and depression (3 patients). Six patients had breast reconstruction, compared to 3 patients in the control group.

Conclusions. The data confirm the increased efficiency of the use of the Multidisciplinary Management File for Breast Cancer Patients, with a better objective and subjective evolution compared to the cases approached in a classical manner. Teamwork facilitates the communication between specialists, as well as between the specialists and the patients.

Keywords: multidisciplinary team, communication, compliance, mastectomy.

\section{Abbreviations}

MMFBCP - Multidisciplinary Management File for Breast Cancer Patients

\section{INTRODUCTION}

Breast cancer is a pathology with increasing incidence: over 2,000,000 new cases of breast cancer were reported ${ }^{1}$ in 2018. The standards used so far provide a unique, individual approach, with the oncologist in the centre of it. The complexity of this pathology, however, makes a multidisciplinary approach ${ }^{2}$ more and more necessary. There is a shortage in the literature regarding the multidisciplinary approach of patients with recommendation for breast reconstruction, and in practice, this approach is almost absent, with few exceptions in some countries. There are standards of approach strictly on the oncological and surgical side 3,4 (including the postsurgical period), but not for the possible needs related to the biomechanical, postural, local biological and psycho-emotional aspects of the patient care (which, in clinical practice, have proven to have a great impact on the results of the whole therapeutic approach). However, in order to cover all these aspects of the rehabilitation, the intervention of several medical and support specialties is necessary, simultaneously or successively.

The multidisciplinary approach is necessary in each stage of the management of an oncological case, from diagnosis to therapy and then breast reconstruction, the result being a higher survival rate $^{5,6}$, the increase of the quality of life ${ }^{7,8}$ and the patient's life expectancy. Working in a team facilitates communication between specialists, on the
Résultats. L'ouverture à l'approche multidisciplinaire a été accrue chez les personnes vivant en milieu urbain ( $80 \%$ dans le groupe d'étude) ainsi que dans l'enseignement supérieur $(90 \%$ diplômés du secondaire ou plus). La présence de pathologies inconnues a été dépistée : 3 pathologies thyroïdiennes, 1 cas de diabète sucré, 6 patientes avec changements de posture, altérations biomécaniques de la ceinture scapulaire, compensations musculaires, anxiété (100\%) et dépression (3 patientes). 6 patientes du groupe d'étude ont eu une reconstruction mammaire, par rapport à 3 patientes du groupe témoin.

Conclusions. Les données confirment l'efficacité accrue de l'utilisation de la Carte de gestion multidisciplinaire pour les patientes atteintes d'un cancer du sein, utilisée avec le groupe d'étude, à une meilleure évolution objective et subjective par rapport aux cas abordés de manière classique. Le travail en équipe facilite la communication entre les spécialistes, ainsi qu'entre les spécialistes et les patients.

Mots-clés: équipe multidisciplinaire, communication, compliance, mastectomie

one hand, by adopting a common therapeutic point of view and conduct, and regulating the timing of the therapeutic acts, avoiding the extension of time for some investigations. Thus, a common standard of therapy is reached, with standards and protocols belonging to different specialties being agreed. On the other hand, working in a multidisciplinary team favours a better communication between specialists and the patient - she gets more congruent, unitary information, which helps to understand more accurately her medical situation and the options she has and, implicitly, to the decrease of the associated anxiety, the end result being an increased treatment compliance and an improvement of the quality of life.

When it comes to a multidisciplinary team, it is often composed of specialists in oncology, oncological surgery, imaging, anatomic pathology and radiotherapy ${ }^{9}$, in a few cases psychiatry or plastic surgery and only in very rare cases other specialties.

The idea of this study is based on a 5-year observation period in centres such as the Cluj-Napoca County Emergency Clinical Hospital - the Plastic Surgery and Reconstructive Microsurgery Department, the AKH Hospital and the Dobling Private Clinic in Vienna (Austria) and the Andarai Hospital from Rio de Janeiro (Brazil), where the authors had the opportunity to observe, comparatively, the approach of the breast cancer cases, from the stage of diagnosis to that of surgical reconstruction. 
The objective of the STUDY was to analyse the opportunity to implement the Multidisciplinary Management File for Breast Cancer Patients (hereinafter referred to as MMFBCP - Multidisciplinary Management File), that includes the investigations, results, observations and recommendations made by all the specialists involved in the management of this type of case. The expected result is the achievement of a more comprehensive management of the breast cancer case, with the increase of the rate of the post-mastectomy breast reconstruction interventions.

\section{Material AND MEthods}

An initial prospective study for the implementation of the MMFBCP was carried out between September 2017 - August 2019 at the Cluj-Napoca County Emergency Clinical Hospital, Romania. Twenty patients, aged between 32 and 65 years, who were diagnosed with a form of breast cancer, were divided into 2 groups: the study group (10 patients) in which the above mentioned MMFBCP was used and the control group (10 patients), in which the classic approach was applied, with interdisciplinary collaboration, but without using the MMFBCP.

The inclusion criteria were:

- breast pathology suitable for reconstruction

- stage up to $\mathrm{T}_{2} \mathrm{~N}_{0} \mathrm{M}_{0}$.

The exclusion criteria were mammary pathologies unable to be reconstructed and those surgically outdated.

Thus, 6 patients had a diagnosis of breast fibroadenoma (4 of which used the MMFBCP), 5 of breast adenocarcinoma (in 3 patients the MMFBCP was used) and 9 with infiltrative ductal carcinoma (3 of them accepted the use of the MMFBCP).

The two groups were constituted exclusively on the ground of the consent for the use of the MMFBCP.

It has two sections:

Section I includes the demographic data: first and last name, age, marital status, race (given that the incidence of malignant mammary disease is higher in the white race ${ }^{10}$ ), professional status (with observations related to the characteristics of the environment - dominant body posture, stress), risk factors (age, genetic mutations of the BRCA1 and BRCA2 genes, obesity, hormonal therapies) ${ }^{11}$, information about the hobby and leisure (e.g. sports ${ }^{12}$ at what level - occasionally, regularly), any other information considered relevant for a global assessment of the person.

This section is initiated by the General Practitioner but can be supplemented with information obtained by any of the specialists who are part of the multidisciplinary case management team.
Section II is the largest and includes the investigations, observations and recommendations of each specialist involved. The specialties we propose are the following:

- Oncology - evaluates the case and proposes the variant of medical or surgical treatment, after consulting with the pathologist.

- Oncological surgery - elaborates the surgical treatment plan.

- Plastic and reconstructive surgery - intervenes to establish the most optimal way of surgical approach for a concomitant breast reconstruction or subsequent to mastectomy. Establishing the type and moment of reconstruction requires the collaboration between the oncologist - oncologist surgeon - plastic surgeon, thereby achieving an effective personalization of the therapeutic protocol.

- Radiotherapy - starts usually three to four weeks after the mastectomy. It is administered by a radiation oncologist in order to destroy the undetectable cancer cells, thus reducing the risk of recurrence.

- Chemotherapy - a systemic therapy designed to destroy or at least slow down the growth of cancer cells, but in some cases also to shrink a tumour before surgery.

- Medical imaging - intervenes both at the initial evaluation and at the long-term monitoring of the case, regardless of the treatment modality chosen.

- Physical medicine and rehabilitation - examines the patient from the point of view of the musculoskeletal status and aims the reintegration of the patients into an active environment from the point of view of the physical activity ${ }^{13,14}$. Also, during the postsurgical period, it is necessary to perform manual lymphatic drainage and specific exercises for the lymphedema that appears after radiotherapy ${ }^{15}$, which are performed at the indication and under the supervision of the specialist of physical medicine and rehabilitation.

- Anaesthesiology - the presurgical assessment of the global health status for the patients' safety during and after the surgical procedures that will follow.

- Physiotherapy - performs the assessment of the postural system and the biomechanics of the scapular belts, upper limbs ${ }^{16}$, cranio-cervical-mandibular system, the assessment of the already existing compensation mechanisms, as well as of other functions that directly interfere with the tissues interested in the breast pathology. This is necessary because the physiotherapist will also intervene in the postsurgical rehabilitation process ${ }^{17}$ and a neglected postural disorder may interfere with recovery and may be misinterpreted as a surgical failure due to breast reconstruction or a postsurgical complication. After mastectomy, there is a tendency to 
decrease physical activity, but also to increase hyper kyphosis. Difficulties arise in performing shoulder movements, as a result of decreased activity of the upper limb, due to lymphedema (which induces or accentuates postural alterations, asymmetries), but also the fear of engaging in movement. Without the specific presurgical assessment (which becomes an integral part of the MMFBCP), we cannot accurately determine the difference between a pre-existing disorder and a postsurgical change. The secondary purpose of this assessment is to inform patients about possible postural changes and their management options.

- Clinical psychology - assesses the patient's cognitive, behavioural, emotional and social status, autonomy in daily and work activities, problem solving ability, decision making, self-esteem, responsibility and accountability, communication, lifestyle, social relationships and the perception of social support ${ }^{18}$, beliefs, emotions. The presurgical counselling the patient will benefit from will focus on accepting the diagnosis, seeking resources and solutions, increasing treatment compliance (both in the case of mastectomy and the breast reconstruction), but also preparing for an uncertain future. The psychologist will monitor the patient also during the postsurgical period (both oncology and post-breast reconstruction), because rehabilitation ${ }^{19}$ does not only mean eliminating the pathology and then prosthesis, but also teaching the patient to live with the change produced, to accept and integrate it (not only physically but especially psychically). Proper and complete rehabilitation leads, in the long-term, to reducing the medical, social and emotional costs associated with the new health state. If there are suspicions ${ }^{20}$, the psychologist will recommend a psychiatric consultation. Also, during the post-mastectomy and post-breast reconstruction period, the patient is strongly advised to attend support groups.

- Endocrinology - evaluates the endocrine status, performs pre- and postsurgical endocrine adjustments. ${ }^{21}$

- Nutrition and diabetes - a specialty that assesses the patient's health and nutrition style, in order to determine possible risk factors and to acquire principles of proper, healthy nutrition ${ }^{22,23}$

- Any other medical or support specialty that can contribute, directly or indirectly, to the accuracy of the diagnosis, the optimal development of the therapeutic plan and to the recovery and rehabilitation of the patient, together with adopting an appropriate lifestyle that will lead to the improvement of the quality of life.

\section{Results}

The patients who accepted the innovative approach of multidisciplinary collaboration based on the MMFBCP came mainly from the urban area $(8$ out of 10); those who refused it came mainly from the rural area ( 7 out of 10 ). The explanation could be the increased accessibility and the greater mobility of the patients in the urban area to all the medical specialties that appear in the file and are compulsory, on one hand, but also the denial of the diagnosis and the fear of the complexity of the file, on the other hand.

As for the academic education:

- in the study group: 4 are university graduates, 2 are post-secondary school graduates, 3 are high school graduates, 1 is a professional school graduate.

- the distribution in the control group is the following: 2 university graduates, 4 high school graduates, 1 professional school graduate, 3 patients have 8 classes or less.

A volunteer was also involved in the study; she was available on the phone for the patients who accepted the MMFBCP and provided assistance, including programming to team member specialists.

The patients of the two groups were assessed by the following medical specialists (as noted in the file, in this order); the results are given in percentage for each group:

1. General Practitioner: $100 \%$ (both groups)

2. Oncologist: $100 \%$ (both groups)

3. Medical imaging: $100 \%$ (both groups)

Laboratory: $100 \%-3$ patients with a family oncological history underwent genetic testing, too.

4. Oncological surgery: 100\% (both groups)

Gynaecology: 100\% (study group) and 40\% (control group) - for specialized consultation, hormonal profile and Pap test

Diabetes, nutrition and metabolic diseases: $100 \%$ of the study group and 20\% of the control group Endocrinology: $100 \%$ of the study group and 0 of the control group.

Physical medicine and rehabilitation + Physiotherapy: 100\% (in the study group), 0 (control group)

Clinical psychology: 100\% (study group) and 0 (control group)

Psychiatry: 100\% study group, 0 control group.

5. Anaesthesia: 100\% (both study and control group)

6. Pathological anatomy: $100 \%$ (both groups)

7. Radiotherapy and Chemotherapy: all patients with infiltrative ductal carcinoma (9, of whom 3 accepted the use of the MMFBCP) had postsurgical radiotherapy. All patients underwent chemotherapy treatment. 
8. Plastic and reconstructive surgery: 8 patients from the study group presented themselves before the mastectomy, for the elaboration of a therapeutic plan for post-mastectomy reconstruction (80\%). Subsequently, 6 of them presented for reconstruction, the 2 excluded being a patient with uncontrolled cardiac pathology and one with diabetes). Of the control group, only 3 were presented at the plastic and reconstructive surgery consultation and subsequently at the breast reconstruction intervention (30\%).

Following the analysis of the centralized data, the following aspects were found:

-3 of the 10 patients who presented to the endocrinological assessment (as part of the MMFBCP) had a thyroid pathology. We cannot anticipate the impact that an undiagnosed thyroid pathology could have on the subsequent evolution of the cases;

- one patient in the study group (10\%) and 2 patients in the control group (20\%) who presented to a nutrition and diabetes assessment were diagnosed with diabetes mellitus (1), respectively with metabolic syndrome (2);

- 6 of the 10 patients who presented to Physical medicine and rehabilitation, respectively Physiotherapy, presented postural changes, alterations of the biomechanics of the scapular belt and mechanisms of muscular compensation

- all the patients who underwent the psychological evaluation had symptoms of anxiety of different intensities, and 3 patients were detected with symptoms of depression

- one of the patients in the study group benefited from psychiatric medication for moderate depression.

- the physical recovery after the mastectomy and breast reconstruction interventions was faster, the patients benefiting from the support of the physiotherapist

- psychological counselling sessions (on average 3 before mastectomy, including assessment, 4 after mastectomy and before breast reconstruction) and group sessions ( 3 sessions, with variable number of participants) contributed to the increase of understanding and acceptance, to increased confidence in medical records and, as a consequence, a better post-mastectomy and breast reconstruction therapeutic compliance.

\section{Discussion}

At first glance, it seems a cumbersome process, with many specialists involved. But a study based on literature search (27 studies included) reports a team members number between 2 and 12. Our maximum number is 14 . In usual care, the reported number of members is 1 or $2 .{ }^{24}$

The main obstacles that may arise in the implementation of this process and, implicitly, of the use of MMFBCP are human, first and foremost:

- finding qualified staff willing to work in a team, as teamwork means a decision to work together, trust in each other and commitment for a shared objective (giving up, to some extent, the autonomy) $)^{25}$. Of course, there is a team leader, case manager, usually the oncologist, but its members are equal in importance in the therapeutic process, each of them has equal access to all the information contained in the file and may complete it, along the way, with any other information considered relevant to the case.

- poor communication between team in members;

- the ambiguity of the role of each member, with an impact on assuming responsibility in the therapeutic approach;

- longer time spent in the process of evaluating the case - in fact, we suggest that all assessments be performed within the usual, "standard" range of time, between the moment of diagnosis and the time of mastectomy; setting up a stable, synchronized multidisciplinary team will surely remove this fear;

- seemingly higher costs - because of involving a big number of specialists in a short time; but the costs can be even higher when omitting some steps or in case of avoidable or minimizable complications;

- the patient's perception - anxious people may consider that their case is extremely serious and that is why they require such a big number of specialists.

Beyond these limitations or obstacles, the benefits are many more:

- early identification of all risk factors that may impede the patient's efficient collaboration with the medical and support staff or can generate true pathologies when another triggering factor occurs ${ }^{26}$.

- increased treatment compliance (whatever it may be) - the patient becomes aware of everything her case involves and can have an overview that helps her to "structure" her near or medium-term future, always knowing what the next step will be in the evolution of the case; the patient's satisfaction is greater in a team-based care delivery, as it can enhance the quality of care ${ }^{27}$.

- choosing the most appropriate treatment option, by consulting and comparing several variants belonging to different medical specialties, based on diagnostic imaging and pathology $y^{28}$;

- choosing the optimal surgical variant for the subsequent accomplishment of breast reconstruction;

- lowering the stress level by providing a secure framework; 
- the reduction of the associated costs, in the long-term, including the social ones, through a quick and efficient social and professional reintegration;

- prevention and, if occurring, early rehabilitation of musculoskeletal complications;

- prevention of the associated psycho-emotional disorders and, subsequently, the breast reconstruction;

- increasing the quality of life and life expectancy ${ }^{29}$.

There is a great amount of literature on the benefits of the teamwork, several studies underlining that the best decision about a possible diagnosis and also for the treatment plan is made in a multidisciplinary team ${ }^{30.32}$; even an assessment tool for the cancer care multidisciplinary team has been developed, with the aim of self-improvement and monitoring. ${ }^{33}$

The LIMITS OF THE STUDY. The main limitation of the study is the small number of patients. Even so, taken as a percentage, the results are promising. An argument for the small number of patients who make up the study group is that the decision of the approach belonged to the medical staff involved and was not an "institutional" one, in other words there is no standard protocol for this approach. Providing organizational support was claimed necessary for the high quality and patient-centred medical care even in countries with a better background in medical teamwork..$^{34}$

\section{Conclusions}

Increasing incidence and complexity of breast cancer requires the extension of diagnostic and treatment options. The approach standards used so far strictly refer to the oncological and surgical evaluation and therapy, taking into account too little the possible needs related to the biomechanical, postural, biological and psycho-emotional aspects related to the patients (which, in clinical practice, have been proven to have a very large impact on the results of the whole therapeutic approach). However, in order to cover all these aspects of the rehabilitation, it is necessary the simultaneous or successive intervention of several medical and support specialties.

Teamwork facilitates the communication between specialists, by adopting a common therapeutic point of view and conduct and synchronizing the therapeutic acts, avoiding the extension of time for some investigations. Thus, a common standard of therapy is reached, with standards and protocols belonging to different specialties being agreed.

On the other hand, multidisciplinary teamwork promotes better communication between specialists and patients, thus obtaining congruent, unitary information, which helps them to understand more precisely their medical situation and the options they have and, implicitly, to reduce the associated anxiety, the final result being the increase of the treatment compliance and the improvement of the quality of life.

Patients who have agreed to comply with the requirements imposed by the Multidisciplinary Management File for Breast Cancer Patients had a better objective and subjective evolution compared to the cases approached in a classical manner.

\section{Author contributions}

Conceptualization, A.C.B., D.D., M.S.P; methodology, A.C.B. and O.A.M.; software, O.A.M.; validation, A.C.B. and D.D.; formal analysis, O.A.M.; investigation, A.C.B., D.D., M.S.P.; resources, A.C.B., D.D., M.S.P.; data curation, A.C.B., D.D., M.S.P.; writing - original draft preparation, A.C.B., D.D., M.S.P.; writing - review and editing, A.C.B. and D.D.; visualization, A.C.B., D.D., M.S.P., O.A.M.; supervision, A.C.B.; project administration, A.C.B., D.D., M.S.P. All the authors have read and agreed with the final version of the article.

\section{Compliance with Ethics Requirements:}

„The authors declare no conflict of interest regarding this article"

"The authors declare that all the procedures and experiments of this study respect the ethical standards in the Helsinki Declaration of 1975, as revised in 2008(5), as well as the national law. Informed consent was obtained from all the patients included in the study"

"No funding for this study"

\section{Acknowledgments: none}

\section{References}

1. Bray F, Ferlay J, Soerjomataram I, Siegel RL, Torre LA, Jemal A. Global cancer statistics 2018: GLOBOCAN estimates of incidence and mortality worldwide for 36 cancers and 185 countries. CA Cancer J Clin. 2018; 68(6):394-424

2. Leclerc AF, Jerusalem G, Devos M, Crielaard JM, Maquet D. Multidisciplinary management of breast cancer. Archives of Public Health. 2016; 74:50

3. Wright FC, De Vito C, Langer B, Hunter A, the Expert Panel on the Multidisciplinary Cancer Conference Standards. Multidisciplinary cancer conference standards. Toronto (ON): Cancer Care Ontario; 2006 Jun 1. Program in Evidence-based Care Evidence-based Series MCC Standards Special Report.

4. Chang JH, Vines E, Bertsch H, et al. The impact of a multidisciplinary breast cancer center on recommendations for 
patient management: the University of Pennsylvania experience. Cancer. 2001; 91:1231-1237.

5. Kesson EM, Allardice GM, George WD, Burns HJG, Morrison DS. Effects of multidisciplinary team working on breast cancer survival: retrospective, comparative, interventional cohort study of 13722 women. BMJ. 2012; 344:e2718.

6. Gilis CR, Hole DJ. Survival outcome of care by specialist surgeons in breast cancer: a study of 3786 patients in the west of Scotland. BMJ. 1996;312:145-8.

7. Engel J, Kerr J, Schlesinger-Raab A, Sauer H, Hölzel D. Quality of life following breast-conserving therapy or mastectomy: results of a 5-year prospective study. Breast J. 2004; 10(3): 223-231

8. Pusic AL, Matros E, Fine N, et al. Patient-reported outcomes 1 year after immediate breast reconstruction: results of the Mastectomy Reconstruction Outcomes Consortium Study. J Clin Oncol. 2017; 35(22):2499-2506.

9. Chae BJ, Bae JS, Song BJ, Jung SS. Multidisciplinary team approach in breast cancer: a nationwide survey in Korea. J Korean Surg Soc. 2012; 82:340-346.

10. Abdulrahman GO, Rahman GA. Epidemiology of breast cancer in Europe and Africa. J Cancer Epidemiol. 2012:915610.

11. Coughlin SS. Epidemiology of breast cancer in women. Adv Exp Med Biol. 2019; 1152:9-29.

12. Lynch BM, Neilson HK, Friedenreich CM. Physical activity and breast cancer prevention. Recent Results Cancer Res. 2011; 186:13-42

13. Herrero F, San Juan AF, Fleck SJ, et al. Combined aerobic and resistance training in breast cancer survivors: A randomized, controlled pilot trial. Int J Sports Med. 2006 27(7):573-80.

14. Daley AJ, Crank H, Saxton JM, Mutrie N, Coleman R, Roalfe A. Randomized trial of exercise therapy in women treated for breast cancer. J Clin Oncol. 2007; 25(13):1713-21.

15. Ezzo J, Manheimer E, McNeely ML, et al. Manual lymphatic drainage for lymphedema following breast cancer treatment. Cochrane Database Syst Rev. 2015;(5):CD003475.

16. Hadad CAS, Saad M, Janeiro Perez MC, Miranda F. Assessment of posture and joint movements of the upper limbs of patients after mastectomy and lymphadenectomy. Einstein (Sao Paulo). 2013; 11(4):426-434.

17. Mangone M, Bernetti A, Agostini F, et al. Changes in spine alignment and postural balance after breast cancer surgery: a rehabilitative point of view. Biores Open Access. 2019; 8(1):121-128

18. Loibl S, Lederer B. The importance of supportive care in breast cancer patients. Breast Care (Basel). 2014; 9(4):230-231.

19. Ganz PA, Yip CH, Gralow JR, et al. Supportive care after curative treatment for breast cancer (survivorship care): resource allocations in low- and middle-income countries. A Breast Health Global Initiative 2013 consensus statement. Breast. 2013; 22(5):606-15.
20. Wefel JS, Vardy J, Ahles T, Schagen SB. International Cognition and Cancer Task Force recommendations to harmonise studies of cognitive function in patients with cancer. Lancet Oncol. 2011; 12(7):703-8.

21. Søgaard M, Farkas DK, Ehrenstein V, Jørgensen JOL, Dekkers OM, Sørensen HT. Hypothyroidism and hyperthyroidism and breast cancer risk: a nationwide cohort study. European Journal of Endocrinology. 2016; 174(4):409-414.

22. Wiseman M. The Second World Cancer Research Fund/ American Institute for Cancer Research Expert Report. Food, Nutrition, Physical Activity and the Prevention of Cancer: A Global Perspective. Nutrition Society and BAPEN Medical Symposium on Nutrition Support in cancer therapy, Proceedings of the Nutrition Society. 2008;67(3):253-256.

23. Howell A, Anderson AS, Clarke RB, et al. Risk determination and prevention of breast cancer. Breast Cancer Research. 2014; 16:446.

24. Wen J, Schulman KA. Can team-based care improve patient satisfaction? A systematic review of randomized controlled trials. PLoS ONE. 2014; 9(7): e100603.

25. Clements D, Dault M, Priest A. Effective teamwork in healthcare: research and reality. Healthcare Papers. 2007; 7 Special Issue:26-34.

26. Manser T. Teamwork and patient safety in dynamic domains of healthcare: a review of the literature. Acta Anaesthesiol Scand. 2009; 53(2):143-151.

27. Litten G, Kane D, Clay G, Kruger P, Belnap T, Parkinson B. Multidisciplinary cancer care with a patient and a physician satisfaction focus. J Oncol Pract. 2010; 6(6): e35-7.

28. Devitt B, Philip J, McLachlan SA. Team dynamics, decision making, and attitudes toward multidisciplinary cancer meetings: health professionals’ perspectives. J Oncol Pract. 2010; 6(6): e17-20.

29. Taylor C, Shewbridge A, Harris J, Green JS. Benefits of multidisciplinary teamwork in the management of breast cancer. Breast Cancer: Targets and Therapy. 2013; 5:79-85.

30. Mogoanta SS, Costache A, Mutiu G., et al. A nonfunctional neuroendocrine tumor of the pancreas - a case report. Romanian Journal of Morphology and Embriology. 2015;56(2 Suppl.):511-519.

31. Ginghina $\mathrm{O}$, Negrei C, Hudita A, et al. In vitro impact of some natural compounds on HT-29 colorectal adenocarcinoma cells. Farmacia. 2017;65(6):947-953.

32. Bratu OG, Cherciu AI, Bumbu A, et al. Retroperitoneal tumors - treatment and prognosis of tumor recurrence. Rev Chim (Bucharest). 2019;70(1):191-194.

33. Lamb BW, Wong HWL, Vincent C, Green JSA, Sevdalis N. Teamwork and team performance in multidisciplinary cancer teams: development and evaluation of an observational assessment tool. BMJ Qual Saf. 2011; 20:849-856.

34. Lamb BW, Taylor C, Lamb JN, et al. Facilitators and barriers to teamworking and patient centeredness in multidisciplinary cancer teams: findings of a national study. Ann Surg Oncol. 2013; 20(5):1408-1416. 